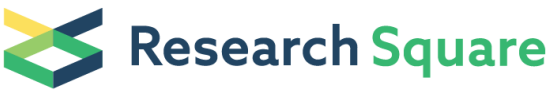 \\ Preprints are preliminary reports that have not undergone peer review. \\ They should not be considered conclusive, used to inform clinical practice, \\ or referenced by the media as validated information.
}

\section{Hemogram Rates as Prognostic Markers of ICU Admission in COVID-}

19

Sara Velazquez

Hospital Universitario HM Sanchinarro

Rodrigo Madurga

Fundación de Investigación HM Hospitales

Jose Maria Castellano

Hospital Universitario HM Montepríncipe

Jesus Rodriguez-Pascual

HM-CIOCC. Hospital Universitario HM Puerta del Sur. Móstoles

Santiago Ruiz de Aguiar

Hospital Universitario HM Puerta del Sur. Móstoles

Sara Jimeno

Hospital Universitario HM Puerta del Sur. Móstoles

Juan Ignacio Montero

Hospital Universitario HM Puerta del Sur. Móstoles

Paula Sol Ventura

Hospital Universitario HM Nens

Alejandro López-Escobar ( $\square$ alopezescobar@hmhospitales.com )

Hospital Universitario HM Puerta del Sur. Móstoles

\section{Research Article}

Keywords: COVID-19, hemogram rates, neutrophil-to-platelet ratio, neutrophil-to-lymphocyte ratio, ICU

Posted Date: April 22nd, 2021

DOI: https://doi.org/10.21203/rs.3.rs-403472/v1

License: (c) (i) This work is licensed under a Creative Commons Attribution 4.0 International License. Read Full License

Version of Record: A version of this preprint was published at BMC Emergency Medicine on July 27th, 2021. See the published version at https://doi.org/10.1186/s12873-021-00480-w. 


\section{Abstract}

\section{Background}

Since first cases of SARS COV-2 were identified, the number of affected and dead people make necessary to identify factors related to worse evolution. Endothelial injury has been proposed as the main pathophysiological mechanism in the illness development, which provokes a hyper inflammation and prothrombotic state. Leukocytes and platelets play a role in inflammation and thrombogenesis so we propose to study if neutrophil-to-lymphocyte ratio (NLR), platelets-to-lymphocyte ratio (PLR), the novel neutrophil-to-platelet ratio (NPR), and the systemic immuneinflammation index (SII), could be useful to identify patients who will need admission at Intensive Care Units.

\section{Methods}

A retrospective observational study was performed at HM Hospitales including 2245 patients with COVID-19 from March 1 to June $10,2020$. Patients were divided into two groups, admitted, and not admitted to ICU.

Results

Patients requiring ICU admission had significantly higher rates at the moment of hospital admission in NLR (6.9 [4-11.7] vs 4.1 [2.6-7.6], p<0.0001), PLR (2 [1.4-3.3] vs 1.9 [1.3-2.9], p=0.023), NPR (3 [2.1-4.2] vs 2.3 [1.6-3.2], p<0.0001) and SII (13 [6.5-25.7] vs 9 [4.9-17.5], $\mathrm{p}<0.0001)$ than those who did not enter ICU. After multivariable logistic regression models, the hemogram rate that better remains as a predictor of ICU admission, when adjusted for the more complex model, was NPR, OR 1.11 (95\% Cl: 0.98-1.22, p=0.055).

Conclusions

The four rates obtained from hemogram at hospital admission, especially the novelty NPR, have shown to be useful as predictors of unfavorable clinical evolution.

\section{Background}

In December 2019, the first cases of pneumonia of unknown cause emerged in the city of Wuhan, China. On January 7th a new coronavirus was identified at the Chinese Center for Disease Control and Prevention as the causative agent of pneumonia. A betacoronavirus (1) was named by the World Health Organization (WHO) as 2019-n-CoV (2). The syndrome developed is known as severe acute respiratory syndrome coronavirus 2 (SARS.CoV-2) (3-5) and on March 11, 2020, with 108.000 cases reported in 114 countries around the world and a total of 4.291 people died, the WHO declared that the outbreak of this disease was considered as a pandemic (6).

Approximately $80 \%$ of infected people have mild to moderate symptoms while the remaining $20 \%$ have a severe clinical presentation. The Intensive Care Unit (ICU) admission differs from 5-32\% (7-9) due to an acute hypoxemic respiratory failure.

The cause of respiratory failure is the development of Acute Respiratory Distress Syndrome (ARDS). It is a diffuse pulmonary inflammatory injury that leads to an increase in pulmonary vascular permeability and makes gas exchange difficult (10). Pathophysiological theories suggest a crucial role of endothelial dysfunction related to evolution of this infectious disease. Endothelial cells are involved in keeping homeostasis, vasomotor tone regulation, vascular permeability, vascular barrier function, and blood rheology $(11,12)$. Endothelial injury may activate, through a cytokine release, a hyperinflammatory and procoagulant state. White blood cells, neutrophils, lymphocytes and monocytes, are directly involved in this systemic inflammatory response while platelets are the main mediators of hemostasis.

It has been described as common laboratory abnormalities in patients with COVID-19 infection, such as increased white blood cells count and neutrophil count and decreased lymphocyte count (13). Hemogram rates such as neutrophil-to-lymphocyte ratio (NLR) and platelet-tolymphocyte ratio (PLR) have been proposed to assess the extension of systemic inflammation.

NLR has been proposed as a prognostic marker of severity in oncological processes (14-16), appears to be an indicator of endothelial dysfunction and an important predictor of cardiovascular mortality $(17,18)$ as well as a mortality predictor in patients with the acute respiratory syndrome $(19,20)$. NLR is associated with worse outcome in patients with SARS-Cov-2 infection (21-23).

PLR has been described as a maker in acute inflammatory and prothrombotic states as well as PLR may reflect the degree of cytokine release, for this reason this ratio might provide useful information about clinical evolution of COVID-19 patients (24).

Systemic immune-inflammation-index (SII), is also a hemogram ratio studied as a prognostic indicator in the follow-up of sepsis (25) and in cancer patients $(26,27)$. 
In this study we propose to include the new neutrophil-to-platelet ratio (NPR). It incorporates the number of neutrophils, implicated in the inflammation response to infection and also involved in the thrombotic mechanism, and a number of platelets whose role in hemostasis is essential as well as his immunomodulatory properties (28).

According to our results that have been published previously (21-23), the main objective of this research was to clear out the role of hemogram rates as prognostic markers of severe COVID-19 defined as the need for ICU admission and as secondary outcomes to find other variables and parameters related to the risk of ICU admission in patients affected by COVID-19.

\section{Materials And Methods}

COVID-19 patients that were hospitalized at any of the 10 hospitals of the HM Hospitales group between March 1 and June 10, 2020 , were retrospectively included in the study. Last follow up of the clinical or laboratory data of the patients included was on June $24,2020$.

A total of 2543 COVID-19 patients were admitted during the study period. Patients whose age was under 18 years old ( $n=5)$, having missing laboratory data in the first 24 hours of admission $(n=258)$ or died at hospital admission $(n=26)$ were excluded. Therefore, from the 2543 patients, 2254 (88.6\%) were included in the final analyses as shown in the flow diagram in Figure S5.

During period previously described, due to the dramatic pandemic situation with a multitude of admitted patients and a shortage of PCR tests, there were changes in the diagnostic protocol by the Spanish Ministry of Health. For several weeks, the diagnosis of Covid-19 was based solely on clinical characteristics and radiological criteria. Infection by SARS-CoV-2 was confirmed by PCR in 2114 (93.8\%) patients. The remaining 140 patients included presented clinical and/or radiological signs compatible with COVID-19, as per protocol.

The study protocol was approved by the HM Hospitales Ethics Committee on March 25, 2020 (approval number 20.03.1573-GHM).

Demographic data, comorbidities, epidemiological characteristics and laboratory findings from each patient were collected from the electronic health report system at hospital admission. All patients were assessed in the emergency department where a blood sample was drawn. Laboratory assessments consisted of complete blood count (including white blood cell count, leukocyte subtypes, hemoglobin count and platelet count), biochemical parameters and blood coagulation tests (D-dimer, prothrombin time and activated partial prothrombin time).

Counts of neutrophils ( $x 10^{9}$ cells $\left./ \mathrm{L}\right)$, lymphocytes ( $x 10^{9}$ cells/L) and platelets $\left(x 10^{11}\right.$ cells/L) were used to define four different inflammatory rates: NLR is the ratio between neutrophils and lymphocytes, PLR is the ratio between platelets and lymphocytes, NPR is the ratio between neutrophils and platelets and, finally, SII is defined as neutrophils multiplied by platelets and divided by lymphocytes.

Moreover, we propose to study the inflammatory rates and their rate of change, getting data from up to four consecutive blood laboratory tests since the patient was admitted to the hospital. The rate of change compares the evolution of NLR, PLR, NPR and SII with the value obtained at admission and was defined as the slope of the linear fit of the relative rates versus time from hospital entry in days. It is considered positive change if the value of the ratio is increased more than $10 \%$ per day, negative if the value decreased at least $10 \%$ per day and in the cases were the change was between $-10 \%$ and $10 \%$ per day were classified as null.

Summary statistics were made for the entire cohort and the patients who needed to be admitted to the ICU and those who were not were grouped together. Continuous variables were summarized as median (interquartile range) and categorical variables as absolute frequency (relative frequency, \%).

Differences between groups were evaluated applying Mann-Whitney $U$ test for quantitative variables and $\mathrm{X}^{2}$ test or Fisher's exact test for categorical variables. Spearman's rho test was used to evaluate correlation between continuous variables. Correlation plots were constructed using the R package GGally.

Variables that demonstrated differences between both groups, admitted and not admitted to ICU, with $\mathrm{p}$ value $<0.2$ were selected for univariable logistic regression.

To implement the bivariable logistic regression models the inflammatory rates were conflated to other variables. Those parameters that added to the model, got modify the inflammatory rates estimate by at least 10 per cent, were considered to build the multivariable adjusted models. Model A included age, heart rate, temperature $>38^{\circ} \mathrm{C}$, systolic and diastolic blood pressure, NLR rate of change $>10 \%$ per day, AST, D-Dimer and glucose. Model B-D included previous model and oxygen saturation (>94, 90-94 or < 90 \%), LDH and CRP respectively (Table 5).

Interaction and stratified analyses were performed for each inflammatory rate adjusted to model A and conducted for age ( $<70$ and $>70$ years), sex, hypertension, diabetes mellitus, oxygen saturation ( $<90$ and $>90 \%)$ and LDH and CRP both categorized through their respective median values (Figs. 1A, S1B, S1C, S1C). 
Statistical inference was performed using two-tailed test and with type I error rate of 0.05 . All statistical analyses were done using R (version 4.0.0).

\section{Results}

Clinical or laboratory data of 2254 patients admitted to Group HM Hospitales due to COVID-19 infection during the study period, from March 1 to June 10, 2020, are shown. Clinical characteristics are summarized in Table 1 and laboratory findings in Tables 2 and 3 . The median age of patients was 69 [57-80] and 59.5\% were men. At the time of admission, all patients were assessed in the emergency room and a blood sample was taken for current analysis and subsequently all patients received standard approved treatment for COVID-19. Infection by SARS-CoV-2 was confirmed in one thousand nine hundred fifty four (93.8\%) patients.

One hundred eighty five (8.29\%) patients experienced severe acute respiratory failure and were admitted to the ICU and were compared with those who did not require ICU admission. Three hundred thirty one (14.7\%) patients passed away, sixty one (33\%) from the ICU group and two hundred seventy $(13 \%)$ of non-ICU group $(p<0.0001)$.

At the time of hospital admission, clinical differences were observed between patients who were admitted to ICU and those who did not, including sex (74.6\% vs $58.1 \%$ males, odds ratio [OR]: $2.11 ; 95 \% \mathrm{Cl}: 1.51-3 ; \mathrm{p}<0.0001)$, temperature above $38^{\circ} \mathrm{C}(14.1 \%$ vs $6.3 \%$, odds ratio [OR]: 2.77; 95\% Cl: 1.72-4.34; p < 0.0001) and SaO2 (SaO2<90\% 22.7\% vs 13.8\%, odds ratio [OR]: 2.94; 95\% Cl: 1.92-4.48; p < 0.0001). Regarding presence of comorbidities, only hypertension was significantly more frequent among ICU-admitted (43.8\% vs 35.8\%, odds ratio [OR]: $1.41 ; 95 \%$ Cl: $1.04-1.91 ; p=0.026)$ (Table 1).

Table 1

Demographics and Clinical characteristics (\% and median value (interquartile range)). ICU-admitted vs Non-ICU admitted. Abbreviations: ICU, Intensive Care Unit; HBP, High blood pressure; DM,

\begin{tabular}{|c|c|c|c|c|c|c|}
\hline & \multirow{2}{*}{$\begin{array}{l}\text { Total } \\
(n=2254)\end{array}$} & \multirow{2}{*}{$\begin{array}{l}\text { ICU } \\
(n=185)\end{array}$} & \multirow{2}{*}{$\begin{array}{l}\text { Non-ICU } \\
(n=2069)\end{array}$} & \multirow[t]{2}{*}{$P$ value } & \multicolumn{2}{|c|}{ Univariable analysis } \\
\hline & & & & & OR $(95 \% \mathrm{Cl})$ & $P$ value \\
\hline \multicolumn{7}{|c|}{ Demographics characteristics } \\
\hline Age (years) & $69(57-80)$ & $68(61-74)$ & $70(57-81)$ & 0.057 & $0.99(0.98-1.00)$ & 0.18 \\
\hline Male (\%) & $59.5 \%$ & $74.6 \%$ & $58.1 \%$ & $<0.0001$ & $2.11(1.51-3.00)$ & $<0.0001$ \\
\hline \multicolumn{7}{|l|}{ Comorbidities } \\
\hline HBP & $36.4 \%$ & $43.8 \%$ & $35.8 \%$ & 0.032 & $1.41(1.04-1.91)$ & 0.026 \\
\hline DM & $18.1 \%$ & $21.6 \%$ & $17.7 \%$ & 0.21 & NA & NA \\
\hline COPD & $5.7 \%$ & $4.3 \%$ & $5.8 \%$ & 0.52 & NA & NA \\
\hline$C D$ & $11.5 \%$ & $11.9 \%$ & $11.5 \%$ & 0.95 & NA & NA \\
\hline \multicolumn{7}{|l|}{ Clinical Characteristics } \\
\hline Temperature $>38^{\circ} \mathrm{C}(\%)$ & $6.9 \%$ & $14.1 \%$ & $130(6.3 \%)$ & $<0.0001$ & $2.77(1.72-4.34)$ & $<0.0001$ \\
\hline Heart rate (bpm) & $89(78-101)$ & $91(84-101)$ & $89(78-101)$ & 0.10 & NA & NA \\
\hline BP max (mm Hg) & $131(117-146)$ & $130(119-140)$ & $131(117-146)$ & 0.36 & NA & NA \\
\hline $\mathrm{BP} \min (\mathrm{mm} \mathrm{Hg})$ & $76(67-84)$ & $66-83)$ & $76(67-84)$ & 0.56 & NA & NA \\
\hline Sat $02(\%)>94 \%$ & $50.4 \%$ & $29.2 \%$ & $52.2 \%$ & $<0.0001$ & NA & NA \\
\hline Sat $02(\%) 90-94 \%$ & $20.4 \%$ & $21.6 \%$ & $20.3 \%$ & $<0.0001$ & $1.91(1.24-2.91)$ & 0.0029 \\
\hline Sat $02(\%)<90 \%$ & $14.6 \%$ & $22.7 \%$ & $13.8 \%$ & $<0.0001$ & $2.94(1.92-4.48)$ & $<0.0001$ \\
\hline Exitus & $14.7 \%$ & $33 \%$ & $13 \%$ & $<0.0001$ & NA & NA \\
\hline
\end{tabular}

In relation to blood test values, many significant differences were found (Tables 2 and 3 ). Assessing rates relating to different parameters of the blood count, patients requiring ICU admission had significantly higher rates at the time of hospital admission in the NLR (6.9 [4-11.7] vs 4.1 [2.6-7.6], p<0.0001), PLR (2 [1.4-3.3] vs 1.9 [1.3-2.9], p=0.023), NPR (3 [2.1-4.2] vs 2.3 [1.6-3.2], p<0.0001) and SII (13 [6.5-25.7] vs 9 [4.917.5], $\mathrm{p}<0.0001)$ than those who were not admitted in the ICU (Table 3). 
Table 2

Laboratory findings at admission. Median value (interquartile range). *The variable is continuous, the OR is for each increment in a unit. ICUadmitted vs Non-ICU admitted. Abbreviations: : ICU, Intensive Care Unit; MCHC, mean corpuscular hemoglobin concentration; MCV, Mean corpuscular volume; MPV, Mean platelet volume; NLR, neutrophil-lymphocyte ratio; PLR, platelet-lymphocyte ratio; NPR, neutrophil-platelets ratio; SII, systemic immune-inflammation index; AST, Aspartate aminotransferase; ALT; lactate aminotransferase; LDH, lactate dehydrogenase.

\begin{tabular}{|c|c|c|c|c|c|c|}
\hline & \multirow{2}{*}{$\begin{array}{l}\text { Total } \\
\text { (n: 2254) }\end{array}$} & \multirow{2}{*}{$\begin{array}{l}\text { ICU } \\
(n=185)\end{array}$} & \multirow{2}{*}{$\begin{array}{l}\text { Non-ICU } \\
(n=2069)\end{array}$} & \multirow[t]{2}{*}{$p$ value } & \multicolumn{2}{|l|}{ Univariable analysis } \\
\hline & & & & & OR $(95 \% \mathrm{Cl})$ & $P$ value \\
\hline \multicolumn{7}{|l|}{ Laboratory findings } \\
\hline White blood cells $\left(10^{\wedge} 9 / \mathrm{L}\right)$ & $6.6(5.0-8.9)$ & $7.2(5.4-10.3)$ & $6.6(5.0-8.8)$ & 0.0034 & $1.04(1.01-1.08) *$ & 0.012 \\
\hline Red blood cells (10^12/L) & $4.7(4.2-5.0)$ & $4.7(4.2-5.0)$ & $4.7(4.2-5.0)$ & 0.85 & NA & NA \\
\hline Neutrophils (10^9/L) & $4.8(3.3-6.9)$ & $5.7(4.0-8.6)$ & $4.7(3.3-6.7)$ & $<0.0001$ & $1.07(1.03-1.10) *$ & 0.00014 \\
\hline Lymphocytes (10^9/L) & $1.1(0.8-1.5)$ & $0.9(0.6-1.2)$ & $1.1(0.8-1.5)$ & $<0.0001$ & $0.59(0.44-0.78) *$ & 0.00036 \\
\hline Monocytes (10^9/L) & $0.5(0.3-0.7)$ & $0.4(0.3-0.6)$ & $0.5(0.3-0.7)$ & $<0.0001$ & $0.27(0.14-0.48) *$ & $<0.0001$ \\
\hline Platelets $\left(10^{\wedge} 9 / \mathrm{L}\right)$ & $205(159-266)$ & $186(150-240)$ & $208(161-268)$ & 0.0034 & $1.00(1.00-1.00)$ & 0.072 \\
\hline Hemoglobin (g/dL) & $13.8(12.5-14.9)$ & $13.9(12.5-15.1)$ & $13.8(12.5-14.9)$ & 0.37 & NA & NA \\
\hline $\mathrm{MCHC}(\mathrm{g} / \mathrm{dL})$ & $33.7(32.8-34.5)$ & $34.0(33.3-34.8)$ & $33.6(32.7-34.4)$ & $<0.0001$ & $1.27(1.14-1.42)^{\star}$ & $<0.0001$ \\
\hline AST (U/L) & $31.6(22.3-49.2)$ & $43.1(29.0-70.7)$ & $31.0(21.8-47.5)$ & $<0.0001$ & $1.01(1.01-1.01) *$ & $<0.0001$ \\
\hline ALT (U/L) & $25.5(16.1-42.4)$ & $32.0(20.3-49.0)$ & $25.0(15.7-41.4)$ & $<0.0001$ & $1.00(1.00-1.00)$ & 0.095 \\
\hline Creatinine (mg/dL) & $0.9(0.7-1.1)$ & $1.0(0.8-1.2)$ & $0.9(0.7-1.1)$ & 0.0042 & $1.10(0.88-1.31)$ & 0.30 \\
\hline LDH (U/L) & $521(397-677)$ & $675(532-931)$ & $510(390-655)$ & $<0.0001$ & $1.00(1.00-1.00) *$ & $<0.0001$ \\
\hline C-reactive protein (mg/L) & $64(24-131)$ & $117(59-225)$ & $61(22-122)$ & $<0.0001$ & $1.01(1.00-1.01)^{*}$ & $<0.0001$ \\
\hline Urea (mg/dL) & $34.7(26.0-49.4)$ & $36.3(27.8-50.6)$ & $34.5(26.0-49.3)$ & 0.18 & NA & NA \\
\hline Glucose (mg/dL) & $114(100-137)$ & $125(111-150)$ & $113(100-135)$ & $<0.0001$ & $1.01(1.00-1.01) *$ & $<0.0001$ \\
\hline Partial thromboplastin time (s) & $32(30-35)$ & $32(30-34)$ & $32(30-35)$ & 0.18 & NA & NA \\
\hline D-dimer (mg/L) & $1(0-1)$ & $1(1-2)$ & $1(0-1)$ & 0.0032 & $1.03(1.01-1.04) *$ & 0.00030 \\
\hline Prothrombine time (s) & $13.3(12.3-14.5)$ & $13.4(12.6-14.7)$ & $13.2(12.3-14.5)$ & 0.24 & NA & NA \\
\hline
\end{tabular}


Table 3

Hemogram rates findings. Median value. (interquartile range). * The variable is continuous, the OR is for each increment in a unit. ICU-admitted vs Non-ICU admitted. The rate of change of the different inflammation rates was obtained with up to four consecutive blood cells measurements since hospital entry. The rate of change was defined as the slope of the linear fit of the relative rates versus time from hospital entry in days. A rate of change higher than $10 \%$ per day was considered as positive. Abbreviations: ICU, Intensive Care Unit; NLR, neutrophil-lymphocyte ratio; PLR, platelet-lymphocyte ratio; NPR, neutrophilplatelets ratio; SII, systemic immune-inflammation index.

\begin{tabular}{|c|c|c|c|c|c|c|}
\hline & \multirow{2}{*}{$\begin{array}{l}\text { Total } \\
(n=2254)\end{array}$} & \multirow{2}{*}{$\begin{array}{l}\text { ICU } \\
(n=185)\end{array}$} & \multirow{2}{*}{$\begin{array}{l}\text { Non-ICU } \\
(n=2069)\end{array}$} & \multirow[t]{2}{*}{ p value } & \multicolumn{2}{|l|}{ Univariable analysis } \\
\hline & & & & & OR (95\%Cl) & $P$ value \\
\hline \multicolumn{7}{|c|}{ Hemogram rates at admission } \\
\hline NLR & $4.3(2.7-8.0)$ & $6.9(4.0-11.7)$ & $4.1(2.6-7.6)$ & $<0.0001$ & $1.03(1.02-1.05) *$ & $<0.0001$ \\
\hline PLR & $1.9(1.3-2.9)$ & $2.0(1.4-3.3)$ & $1.9(1.3-2.9)$ & 0.023 & $1.13(1.05-1.21)^{*}$ & 0.00057 \\
\hline NPR & $2.3(1.6-3.3)$ & $3.0(2.1-4.2)$ & $2.3(1.6-3.2)$ & $<0.0001$ & $1.15(1.09-1.22) *$ & $<0.0001$ \\
\hline SII & $9.2(5.0-18.2)$ & $13.0(6.5-25.7)$ & $9.0(4.9-17.5)$ & $<0.0001$ & $1.01(1.01-1.02) *$ & 0.00028 \\
\hline \multicolumn{7}{|c|}{ Positive rate of change $\left(>10 \% \cdot\right.$ day $\left.^{\wedge}-1\right)$} \\
\hline NLR & $20.9 \%$ & $37.8 \%$ & $19.4 \%$ & $<0.0001$ & $1.87(1.33-2.65)$ & 0.00036 \\
\hline PLR & $27.2 \%$ & $35.7 \%$ & $26.5 \%$ & 0.33 & NA & NA \\
\hline NPR & $42.7 \%$ & $60 \%$ & $41.1 \%$ & 0.0029 & $1.74(1.26-2.42)$ & 0.00087 \\
\hline SII & $30 \%$ & $44.9 \%$ & $28.7 \%$ & 0.003 & $1.55(1.11-2.19)$ & 0.011 \\
\hline
\end{tabular}

Independent mortality prediction ability was shown for each hemogram-derived ratio (ROC curves are shown in Figure S2 and optimal cut-off values are shown in Table 4).

Table 4

Optimal cut-off values for the different immunoinflammatory ratios with their sensitivities and specificities and their corresponding 95\% confidence interval.

\begin{tabular}{|llll|}
\hline Variable & Cut-off & Sensitivity & Specificity \\
\hline NLR & 4.93 & $0.68(0.49-0.80)$ & $0.58(0.47-0.74$ \\
\hline PLR & 2.50 & $0.47(0.19-0.85)$ & $0.66(0.27-0.89)$ \\
\hline NPR & 2.44 & $0.68(0.54-0.77)$ & $0.58(0.54-0.71)$ \\
\hline SII & 12.26 & $0.55(0.32-0.87)$ & $0.64(0.30-0.84)$ \\
\hline
\end{tabular}

As for the evolution of these rates on successive days, patients requiring ICU admission showed a significantly higher rate of ascent in the velocity of NLR (37.8\% vs $19.4 \%$ odds ratio [OR]: 1.87 ; $95 \%$ Cl: $1.33-2.65, \mathrm{p}<0.0001)$, NPR (60\% vs $41.1 \%$ odds ratio [OR]: 1.74 ; $95 \%$ Cl: $1.26-$ $2.42, p=0.0029)$ and SII (44.9\% vs $28.7 \%$ odds ratio [OR]: $1.55 ; 95 \%$ Cl: $1.11-2.19, p=0.0032)$, but not in the velocity of PLR (35.7\% vs $26.5 \%, p$ $=0.33)($ Table 3$)$.

The results of multivariable logistic regression models assessing the relation of the different hemogram rates and needing of ICU admission are shown in Table 5. Model A adjusted the hemogram rates OR for age, heart rate, temperature $>38^{\circ} \mathrm{C}$, systolic and diastolic blood pressure, NLR rate of change $>10 \%$ per day, AST, D-Dimer and glucose. This adjustment made every inflammatory rate to lose its association with ICU admission but NPR (model A adjusted OR: 1.15, 95\% Cl: $1.05-1.25, \mathrm{p}=0.0018$ ). NPR showed no dependence with $\mathrm{SaO}_{2}<90 \%$ (model $\mathrm{B}$ adjusted OR: $1.14,95 \% \mathrm{Cl}: 1.03-1.25, \mathrm{p}=0.0044)$ nor with LDH (model C adjusted OR: $1.14,95 \% \mathrm{Cl}: 1.03-1.24, \mathrm{p}=0.0061$ ), but did show an adjusted OR drop to 1.11 (95\% Cl: 1.03-1.24) with a border line significance with CRP (model D, $p=0.055)$ (Table 5). 
Table 5

Multivariable adjusted models. Model A: Age, heart rate, temperature $>38^{\circ} \mathrm{C}$, systolic and dyastolic blood pressure, NLR rate of change $>10 \%$ per day, AST, D-dimer and glucose. Model B: Model A+Saturation 02. Model C: Model B + LDH. Model D: Model C + CRP. Abbreviations: NLR, neutrophil-lymphocyte ratio; PLR, platelet-lymphocyte ratio; NPR, neutrophil-platelets ratio; SII, systemic immune-inflammation index; BP, blood pressure; LDH, lactate dehydrogenase; CRP, C-reactive protein.

\begin{tabular}{|llllll|}
\hline Model & & NLR & PLR & NPR & SII \\
\hline Unadjusted & OR $(95 \% \mathrm{Cl})$ & $1.03(1.02-1.05)$ & $1.13(1.05-1.21)$ & $1.15(1.09-1.22)$ & $1.01(1.01-1.02)$ \\
\cline { 2 - 6 } Model A & p value & $<0.0001$ & $\mathbf{0 . 0 0 0 5 7}$ & $<0.0001$ & $\mathbf{0 . 0 0 0 2 8}$ \\
& OR $(95 \% \mathrm{Cl})$ & $1.02(0.993-1.05)$ & $1.04(0.903-1.17)$ & $1.15(1.05-1.25)$ & $1.01(0.995-1.02)$ \\
\hline Model A+SatO2 & OR $(95 \% \mathrm{Cl})$ & $1.02(0.985-1.05)$ & $1.03(0.888-1.18)$ & $1.14(1.03-1.25)$ & $1.01(0.993-1.02)$ \\
\cline { 2 - 6 } & p value & 0.24 & 0.65 & $\mathbf{0 . 0 0 4 4}$ & 0.31 \\
\hline Model B + LDH & OR $(95 \% \mathrm{Cl})$ & $1.02(0.983-1.05)$ & $1.03(0.885-1.17)$ & $1.14(1.03-1.24)$ & $1.01(0.992-1.02)$ \\
\cline { 2 - 6 } & p value & 0.3 & 0.68 & $\mathbf{0 . 0 0 6 1}$ & 0.37 \\
\hline Model C+ CRP & OR $(95 \% \mathrm{Cl})$ & $0.998(0.961-1.03)$ & $0.979(0.831-1.13)$ & $1.11(0.986-1.22)$ & $0.996(0.98-1.01)$ \\
\cline { 2 - 6 } & p value & 0.93 & 0.78 & 0.055 & 0.64 \\
\cline { 2 - 6 }
\end{tabular}

Stratified analysis showed that increasing values of NPR significantly associates with ICU admission for age < 70 years (odds ratio [OR]: 1.34 ; 95\% Cl: 1.1.17-1.56, p < 0.001), sex male (odds ratio [OR]: 1.24; 95\% Cl: 1.10-1.41, p<0.001), absence of hypertension (odds ratio [OR]: 1.22 ; 95\% Cl: 1.07-1.39, p = 0.0025), SatO2 > 90\% (odds ratio [OR]: 1.23; 95\% Cl: 1.11-1.39, p < 0.001), LDH below median (<677 U/L) (odds ratio [OR]: 1.2; 95\% Cl: 1.08-1.34, p<0.001) and CPR below median (<131 mg/L) (odds ratio [OR]: 1.16; 95\% Cl: 1.02-1.31, p = 0.0098), showing statistical significant interaction with age $(p=0.009)$ and $\mathrm{SaO}_{2}(p=0.024)($ Fig. $1 \mathrm{~A})$.

Higher values of NLR were significantly associated with ICU entry only for strata with $\mathrm{SaO}_{2}>90 \%$ (odds ratio [OR]: $1.04 ; 95 \% \mathrm{Cl}: 1.00-1.08, \mathrm{p}=$ 0.03), male sex (odds ratio [OR]: 1.04; 95\% Cl: 1.01-1.07, $\mathrm{p}=0.015$ ) and age < 70 years (odds ratio [OR]: 1.09; 95\% Cl: 1.04-1.14, $\mathrm{p}=<0.001$ ), this last one showing significant interaction $(p=0.06)$ (Figure S1B).

Higher values of SII were significantly associated with ICU entry for patients with CRP lower than 131 mg/L (median value) (odds ratio [OR]: 1.02; 95\% Cl: 1.00-1.04, $\mathrm{p}=0.045$ ) and for patients younger than 70 (odds ratio [OR]: 1.03; 95\% $\mathrm{Cl}: 1.01-1.05, \mathrm{p}=0.0019$ ), with significant interaction found with age $(p=0.009)$ (Figure S1C).

PLR showed no significant association with ICU entry in the stratified analysis (Figure S1D).

When tested the dependency of the variables used for stratification by Chi-squared test we obtained that being older or younger than 70 years is related with sex $(p<0.0001), \mathrm{HBP}$ (high blood pressure) $(p<0.0001), \mathrm{SaO}_{2}<90 \%(p<0.0001), \mathrm{LDH}<677 \mathrm{U} / \mathrm{L}(\mathrm{p}=0.0004)$ and CRP $<131 \mathrm{mg} / \mathrm{L}$ $(p=0.0023)$.

Correlation analysis between the four hemogram ratios (Figure S3) shows that NLR is correlated with the other three independently from mortality (NLR vs PLR, $\rho=0.7, p<0.001$; NLR vs NPR, $\rho=0.669, p<0.001$; NLR vs SII, $\rho=0.894, p<0.001$ ). However, PLR is correlated with SII ( $\rho$ $=0.816, p<0.001)$ but not with NPR $(\rho=0.013, p=0.53)$. Finally, NPR and SIl showed a significant but weak correlation $(\rho=0.424, p<0.001)$ (Figure S3).

As expected, the hemogram ratios were correlated with some of the hemogram parameters like white blood cells (NLR: $\rho=0.536, p<0.001 ;$ NPR, $\rho=0.622, p<0.001 ;$ SII, $\rho=0.642, p<0.001$ ), neutrophils (NLR: $\rho=0.743, p<0.001 ; N P R, \rho=0.722, p<0.001 ; S I I, \rho=0.792, p<0.001$ ), lymphocytes (NLR: $\rho=-0.699, p<0.001$; PLR, $\rho=-0.727, p<0.001 ;$ SII, $\rho=-0.502, p<0.001$ ) or platelets $(S I I, \rho=0.51, p<0.001)$. All the hemogram rates were significantly but weakly correlated with most of the different laboratory and demographic variables but NLR and CRP $(\rho=$ $0.552, \mathrm{p}<0.001$ ) which was the only case with a correlation higher than 0.5 (Figures S4A and S4B).

\section{Discussion}

As the number of patients with COVID-19 infection is rapidly increasing worldwide, early identification of patients at risk of progression to a critical state is essential both to consider an aggressive specific intervention and to identify differential clinical risk groups. 
Several studies have published many different clinical scores trying to predict patients at risk of ICU admission or die (22, 29-31). A recent systematic review by Wynants et al. showed that the vast majority of the published studies presented some relevant limitations, such as a nonrepresentative selection of control patients, lack of sufficient complete follow-up or poor selection of the overall study sample (32).

A large number of parameters recorded have been widely used as prognostic factors in the inpatient setting. In our study, male gender and arterial hypertension were risk factors for ICU admission. These findings are in consonance with previous reported studies. Especially arterial hypertension could reflect a clinical predisposition to vascular damage, typical of some severe cases of COVID-19. However, we did not find an incremented risk in patients presenting other comorbidities in relationship with potential vascular damage as Diabetes Mellitus or Cardiovascular Disease, even though these comorbidities were prevalent in our study population.

Blood test values have been widely used in early evaluation and monitoring of COVID-19 patients. In our study many differences were found in patients whose were admitted at ICU compared to non-ICU admitted including higher levels of LDH, CRP, APTT and D-Dimer. They also presented higher white blood cell count and neutrophils, while the inverse relationship was seen in non-neutrophil white blood cell series (low lymphocyte, monocyte and eosinophil ranges) and platelet levels (Table 2).

Many of these parameters, specifically derived from white blood cell or platelet count values, provide information on both immunological status and hemostasis in response to SARS-CoV-2 infection. In fact, the host's inflammatory response to SARS-CoV-2 infection appears critical in clinical evolution of COVID-19, and blood cell interactions are essential in the pathophysiology of inflammation, immune responses and hemostasis in this setting. These complex interactions need to be clarifying in order to understand the specific roles of each cell type in the different phases of the same disease. White blood cells are responsible for the immune responses while platelets are the main mediators of hemostasis. Accumulating evidence suggests that a part of patients presenting with severe COVID-19 may present an underlying hyperinflammatory response driving a cytokine release storm resulting in multiorgan failure and death (33). This novel form of microvascular obstructive thromboinflammatory syndrome has been proposed as the pathophysiology underlying this hyperinflammatory response.

These hematological findings are in consonance with other published data, however, our study emphasize the utility of combined white cell count ratios in evolution of hospitalized patient. This fact reflects the complexity and heterogeneity of SARS-CoV-2 infection response: not only the total number of white cell, lymphocyte neutrophil or platelet recruited, but the balance between blood cell subtypes provide an adequate or ineffective immune response to SARS-CoV-2 infection.

Our retrospective study shows the utility of four combined hemogram values as useful hospital predictors of unfavorable clinical evolution in a large number of COVID-19 infected patients. Specifically, NRL, PRL, SIl and PLR could be useful combined indicators of the inflammatory and immunological host status.

NLR has been used as inflammatory marker of COVID-19 $(23,24,34-37)$, and its prognostic value stands out among our findings (21,22). NLR could show the importance of the balance between neutrophil and lymphocytes and it seems to be an independent, robust and reproducible parameter in routine clinical practice.

On the other hand, the new NPR emphasizes the importance of the relationship between immune response and homeostasis (21,22). An endothelial thrombo-inflammatory syndrome has been hypothesized following alveolar viral damage by SARS-CoV-2 (38) and other virus agents (39). This would be caused by infection of endothelial cells using the cell surface angiotensin converting enzyme 2 receptor expressed in these cells $(40,41)$ and it has been demonstrated endothelial cell involvement across vascular beds of different organs in necropsies of patients with COVID-19 (42,43). We hypothesize that a damaged and activated endothelium would increase the permeability and release of cytokines that would increase the chemotaxis of inflammatory cells and also send signals to the blood cells to favor the repair. In this context, after this aggression, platelets and neutrophils are activated by soluble agonists and adhesive proteins via their surface receptors playing a determining role in microvascular occlusion during thromboinflammatory disease (44).

NPR amplifies the value of this relation and we propose this rate as a new biomarker that could be useful to predict prognosis and severity of COVID-19 $(14,15,16)$. This ratio has demonstrated to be very useful to predict in hospital mortality $(21,22)$. Due to the relevant role of neutrophils and platelets in SARS-CoV-2 clinical evolution, we postulate that an increase in the value of neutrophils in relation to a decrease in platelets count could be a relevant prognostic factor, especially in early detection of potential severe cases of COVID-19. As we discussed, the role of neutrophils in response to infection and inflammation is well known, and as for platelets, their role in haemostasis is fundamental when vascular damage occurs. However, the role of neutrophils in thrombosis is increasingly recognized and more is known about the immunomodulatory properties of platelets in such a way that they interact with each other during infection, inflammation and thrombosis by modulating the functions of each (14).

On the other hand, the dynamic evolution of the immune response to SARS-CoV-2 infection could be crucial in the evolution of COVID-19 patients. Previous publications have shown the utility of velocity of change of hemogram rates to predict mortality in COVID-19 patients (21,22). According to our results, the velocity of change of four hemogram rates during first days of hospital staying would be signaling a greater 
inflammatory state in some patients who will later require admission to the ICU as these parameters together with rates at hospital admission have shown their usefulness as prognostic markers of inflammation in patients who ultimately required admission to ICU. In this sense, the hemogram is a tool within the reach of all hospitals and doctors who do not have the technical and material means to carry out complex immunological studies, which often produce late results. The analysis of the hemogram's rates would provide much more information than could be extracted a priori by evaluating the parameters in isolation. We now know that it is crucial to initiate early anti-inflammatory treatment when the patient deteriorates and the hemogram could be an indicator of that signal that could indicate which patients could potentially benefit from earlier anti-inflammatory therapy.

Dynamic changes during the evolution of the disease distort the assessment of every therapeutic intervention, especially in retrospective studies. An increase in the frequency of use of a drug due to clinical aggravation may not be distinguished from an effect of that drug. It is well known that the mechanisms of action of corticosteroids translate into several effects on the response of the immune system, classically producing lymphopenia, neutrophilia and also decreasing cytokine production. In theory, this mechanism may explain an increase in the hemogram rates. However, various studies regarding the prognostic value of NLR in inflammatory diseases (for example, in Bechet's disease or Alcoholic hepatitis), have shown a reduction in the ratio in patients under corticosteroid treatment $(45,46)$. In our study, patients admitted to ICU presented significant differences in the use of corticosteroids as a result of clinical worsening after symptom onset. $59.4 \%$ of patients admitted to ICU were treated with corticosteroids versus $44.4 \%$ of non-ICU admitted $(p<0.0001)$, but the group with worst outcome received the treatment later than those with milder form (4 days [1-11] vs 2 (1-4] days, $p<0.0001$ ) (data not shown in tables). While corticosteroid treatment may account for some effect on velocity of change, it should not influence hemogram rates on admission. This approach highlights the need for dynamic assessment of patients, and may clarify the role of uncontrolled and nonrandomized drugs in COVID-19 studies.

Our study presented several limitations. Diagnostic criteria during the first months of the pandemic in Spain changed, from detection by PCR to clinical and radiological criteria as a consequence of changes in the diagnostic protocol set for by the Spanish Ministry of Health amidst the overworked health system. In fact, $6.2 \%$ of patients in our study COVID-19 was suspected. Additionally, the results of the stratified analysis (Figs. 1A, S1B, S1C and S1D) suggest that age is a significant factor that influences on ICU admission, affecting the rest of the variables. This fact could explain the association with mortality of age over 70 years with sex, hypertension, SatO2 >90, LDH > 677 U/L and CRP > 131 mg/L. During the study period, due to the pandemic situation in Spain, there was limited access to ICU beds and ventilators, which could have conditioned some results since patients could have been candidates for ICU based on age, comorbidities and survival chances. However, NPR is the only hemogram rate that maintains predictive capacity in the lower risk strata (< 70 years, male sex, Sat02 >90, LDH > 677 U/L and CRP > $131 \mathrm{mg} / \mathrm{L})$, which implies that their degree of independence with respect to these variables is greater (Fig. 1A). After reviewing our data, the same reason could explain the mortality rate of patients not admitted to the ICU. Although this number is small, it represents a percentage that may be relevant for the final analysis. This data may derive from patients with a high number of comorbidities or extreme age, a priori without ICU admission criteria. The presence in our study of other patients without ICU criteria but with a favorable evolution is not well established, however, this fact reproduces the usual clinical practice at the present time. Similarly, the use of drugs such as corticosteroids, antivirals and other drugs were variable, and did not always respond to the same criteria during the study period. Finally, this is a retrospective study and lacks a control group, which limits the systematic adoption in routine clinical practice. Further comprehensive studies are needed to determine how useful are these blood tests and the rates at which future prognostic scores demonstrate usefulness in guiding treatment decisions

\section{Conclusions}

Our group is reporting the potential use of different blood test rates and their dynamic assessment as prognostic implications for ICU admission risk in patients affected by COVID-19.

Therefore, as the hemogram is a tool within the reach of all hospitals and doctors, we propose the use of the four hemogram rates for the assessment of the patient affected by COVID-19, especially the novelty NPR that could be very useful as a marker in the prognosis of this disease since it includes inflammatory and thrombotic biomarkers, the main mechanisms involved in the development of severe manifestations of COVID-19.

\section{List Of Abbreviations}

ICU: Intensive care Unit

NLR: Neutrophil-to-lymphocyte ratio

PLR: platelet-to-lymphocyte ratio

SII: The systemic inflammation Index

NPR: neutrophil-platelet ratio 
AST: Aspartate aminotransferase

ALT: lactate aminotransferase

LDH, lactate dehydrogenase

CRP: C-reactive protein

HTA: Arterial hypertension

\section{Declarations}

\section{Ethics approval and consent to participate}

All the methods used in study were performed in accordance with the relevant guidelines and regulations.

The study protocol was approved by the HM Hospitales Ethics Committee on March 25, 2020 (approval number 20.03.1573-GHM).

Informed consent was obtained from all subjects as they all agreed to access and analyze their medical story data.

\section{Consent for publication}

Not applicable

Availability of data and materials

The datasets used and/or analysed during the current study are available from the corresponding author on reasonable request.

\section{Conflict of interests/Competing interests}

The authors declare that they have no competing nor conflict of interests.

\section{Funding}

This research did not receive any specific grant from funding agencies in the public, commercial, or not-for-profit sectors.

\section{Author contibutions}

- Designed research/study: 1,2,3,5,9

- Performed research/study: 1,2,3,5,9

- Collected data: 2,3,5

- Analyzed data: 1,2,4,9

- Wrote paper: $1,2,3,4,5,6,7,8,9$

\section{Acknowledgments}

NA

\section{References}

1. Lu R, Zhao X, Li J, Niu P, Yang B, Wu H, et al. Genomic characterization and epidemiology of 2019 novel coronavirus: implications for virus origins and receptor binding. Lancet 2020 -;395(10224):565.

2. WHO. Clinical management of severe acute respiratory infection when Novel coronavirus (nCoV) infection is suspected: interim guidance. Jan 11, 2020. Available on line: URL:

https://www.who.int/internalpublicationsdetail/clinicalmanagementofsevereacuterespiratoryinfectionwhennovelcoronavirus(ncov)infection is suspected (accessed August 25, 2020).

3. Yin Y, Wunderink RG. MERS, SARS and other coronaviruses as causes of pneumonia. Respirology 2018 -;23(2):130.

4. Drosten C, Günther S, Preiser W, et al. Identification of a novel coronavirus in patients with the severe acute respiratory syndrome. $N$ Engl $J$ Med 2003; 348: 1967-76. 
5. Zaki AM, van Boheemen S, Bestebroer TM, Osterhaus AD, Fouchier RA. Isolation of a novel coronavirus from a man with pneumonia in Saudi Arabia. N Engl J Med 2012; 367: 1814-20.

6. Organización Mundial de la Salud. Available on line: URL: https://www.who.int/es/dg/speeches/detail/who- director-general-s-openingremarks-at-the-media-briefing-on-covid-19-11-march-2020 (accesed August 25, 2020).

7. Guan WJ, Ni ZY, Hu Y, et al. Clinical Characteristics of Coronavirus Disease 2019 in China. N Engl J Med. 2020;382(18):1708-1720.

8. Huang C, Wang Y, Li X, et al. Clinical features of patients infected with 2019 novel coronavirus in Wuhan, China. Lancet. 2020;395(10223):497-506.

9. Grasselli G, Zangrillo A, Zanella A, et al. Baseline Characteristics and Outcomes of 1591 Patients Infected With SARS-CoV-2 Admitted to ICUs of the Lombardy Region, Italy. JAMA. 2020;323(16):1574-1581.

10. ARDS Definition Task Force, Ranieri VM, Rubenfeld GD, et al. Acute respiratory distress syndrome: the Berlin Definition. JAMA. 2012;307(23):2526-2533.

11. Roumenina LT, Rayes J, Frimat M, Fremeaux-Bacchi V. Endothelial cells: source, barrier, and target of defensive mediators. Immunol Rev. 2016;274: 307-29. 4.

12. Daniel AE, van Buul JD. Endothelial junction regulation: a prerequisite for leukocytes crossing the vessel wall. J Innate Immun;2013; 5:32435.

13. Słomka A, Kowalewski M, Żekanowska E. Coronavirus Disease 2019 (COVID-19): A Short Review on Hematological Manifestations. Pathogens. 2020 Jun 20;9(6):493.

14. Guthrie GJ, Charles KA, Roxburgh CS, Horgan PG, McMillan DC, Clarke SJ. The systemic inflammation-based neutrophil-lymphocyte ratio: experience in patients with cancer. Crit Rev Oncol 2013;88(1):218-230.

15. Walsh S, Cook E, Goulder F, Justin T, Keeling N. Neutrophil-lymphocyte ratio as a prognostic factor in colorectal cancer. J Surg Oncol 2005;91(3):181-184.

16. Sarraf KM, Belcher E, Raevsky E, Nicholson AG, Goldstraw P, Lim E. Neutrophil/lymphocyte ratio and its association with survival after complete resection in non-small cell lung cancer. J Thorac Cardiovasc Surg 2009;137(2):425-428.

17. Martínez-Urbistondo D, Beltrán A, Beloqui O, Huerta A. El índice neutrófilo/linfocito como marcador de disfunción sistémica endotelial en sujetos asintomáticos. Nefrología (Madrid) 2016,36(4):397-403.

18. Shah N, Parikh V, Patel N, Patel N, Badheka A, Deshmukh A, et al. Neutrophil lymphocyte ratio significantly improves the Framingham risk score in prediction of coronary heart disease mortality: insights from the National Health and Nutrition Examination Survey-III. Int $J$ Cardiol 2014;171(3):390-397.

19. Wang Y, Ju M, Chen C, et al. Neutrophil-to-lymphocyte ratio as a prognostic marker in acute respiratory distress syndrome patients: a retrospective study. J Thorac Dis. 2018;10(1):273-282.

20. Li W, Ai X, Ni Y, Ye Z, Liang Z. The Association Between the Neutrophil-to-Lymphocyte Ratio and Mortality in Patients With Acute Respiratory Distress Syndrome: A Retrospective Cohort Study. Shock. 2019;51(2):161-167.

21. Lopez-Escobar A, Madurga R, Castellano JM et al. Hemogram as maker of in-hospital mortality in COVID-19. J. Investig. Med. 2021. (Accepted).

22. Lopez-Escobar A, Madurga R, Castellano JM et al. Risk score for predicting in-hospital mortality in COVID-19 (RIM Score). Diagnostics. 2021 (accepted).

23. Jimeno S, Ventura PS, Castellano JM, García-Adasme SI, Miranda M, Touza P, Lllana I, López-Escobar A. Prognostic implications of neutrophil-lymphocyte ratio in COVID-19. Eur J Clin Invest. 2021 Jan;51(1):e13404.

24. Qu R, Ling Y, Liu H-, Zhang Y--, Wei L-, Chen X, et al. Platelet-to-lymphocyte ratio is associated with prognosis in patients with coronavirus disease-19. J Med Virol 2020 ; 92(9):1533.

25. Pedersen SF, Ho YC. SARS-CoV-2: a storm is raging. J Clin Invest 2020;130(5):2202.

26. Hu B, Yang XR, Xu Y, et al. Systemic immune-inflammation index predicts prognosis of patients after curative resection for hepatocellular carcinoma. Clin Cancer Res 2014;20(23):6212-6222.

27. Zhang Y, Sun Y, Zhang Q. Prognostic value of the systemic immune-inflammation index in patients with breast cancer: a meta-analysis. Cancer Cell Int 2020;20(1):1-12.

28. Lisman T. Platelet-neutrophil interactions as drivers of inflammatory and thrombotic disease. Cell Tissue Res. 2018,371(3):567-576.

29. Liang W, Liang H, Ou L, Chen A, Li C, Li Y, et al. Development and validation of a clinical risk score to predict the occurrence of critical illness in hospitalized patients with COVID-19. JAMA Internal Medicine 2020 ; 180(8):1081.

30. Gong J, Chen Y, Hu B, Ou J, Tan M, Shi Y, et al. A Tool for Early Prediction of Severe Coronavirus Disease 2019 (COVID-19): A Multicenter Study Using the Risk Nomogram in Wuhan and Guangdong, China. Clinical infectious diseases: an official publication of the Infectious Diseases Society of America 2020 ; 71(15):833.

Page 11/13 
31. Ji D, Zhang D, Chen Z, Zhao P, Chen G, Bi J, et al. Prediction for Progression Risk in Patients with COVID-19 Pneumonia: the CALL Score. Clinical infectious diseases: an official publication of the Infectious Diseases Society of America 2020 -.

32. Wynants L, Smits LJM, Van Calster B, De Vos M, Steyerberg EW, Collins GS, et al. Prediction models for diagnosis and prognosis of covid19: Systematic review and critical appraisal. The BMJ $2020 ; 369$.

33. Mehta P, McAuley DF, Brown M, Sanchez E, Tattersall RS, Manson JJ. COVID-19: consider cytokine storm syndromes and immunosuppression. Lancet (London, England) 2020 -;395(10229):1033.

34. Zeng F, Li L, Huang H, Deng G, Zeng J, Deng Y, et al. Can we predict the severity of coronavirus disease 2019 with a routine blood test? Polish Archives of Internal Medicine $2020 ; 130(5): 400$.

35. Liu Y, Du X, Chen J, Jin Y, Peng L, Wang HHX, et al. Neutrophil-to-lymphocyte ratio as an independent risk factor for mortality in hospitalized patients with COVID-19. J Infect 2020 -;81(1): e6.

36. Liu J, Xiang P, Pu L, Xiong H, Li C, Zhang M, et al. Neutrophil-to-lymphocyte ratio predicts critical illness patients with 2019 coronavirus disease in the early stage. Journal of Translational Medicine $2020 ;$;8(1).

37. Yang A, Liu J, Tao W, Li H. The diagnostic and predictive role of NLR, d-NLR and PLR in COVID-19 patients. Int Immunopharmacol 2020 -;84.

38. Ciceri F, Beretta L, Scandroglio AM, Colombo S, Landoni G, Ruggeri A, et al. Microvascular COVID-19 lung vessels obstructive thromboinflammatory syndrome (MicroCLOTS): An atypical acute respiratory distress syndrome working hypothesis. Critical Care and Resuscitation $2020 ; 22(2): 95$.

39. Yang $\mathrm{Y}$, Tang $\mathrm{H}$. Aberrant coagulation causes a hyper-inflammatory response in severe influenza pneumonia. CELLULAR AND MOLECULAR IMMUNOLOGY $2016-; 13(4): 432$.

40. Maggi E, Canonica GW, Moretta L. COVID-19: Unanswered questions on immune response and pathogenesis. J Allergy Clin Immuno/ 2020 $; 146(1): 18$.

41. Mehra MR, Desai SS, Kuy S, Henry TD, Patel AN. Cardiovascular disease, drug therapy, and mortality in COVID-19. N Eng/ J Med 2020 ;382(25): E102.

42. Monteil V, Kwon H, Prado P, Hagelkrüys A, Wimmer RA, Stahl M, et al. Inhibition of SARS-CoV-2 Infections in Engineered Human Tissues Using Clinical-Grade Soluble Human ACE2. Cell 2020 ; 181(4):905.

43. Varga Z, Flammer AJ, Steiger P, Haberecker M, Andermatt R, Zinkernagel AS, et al. Endothelial cell infection and endotheliitis in COVID-19. Lancet (London, England) 2020 ;395(10234):1417.

44. Li J, Kim K, Barazia A, Tseng A, Cho J. Platelet-neutrophil interactions under thromboinflammatory conditions. Cellular and molecular life sciences $2015 ; 72(14): 2627$.

45. Djaballah-Ider F, Touil-Boukoffa C. Effect of combined colchicine-corticosteroid treatment on neutrophil/lymphocyte ratio: a predictive marker in Behçet disease activity. Inflammopharmacology 2020 -;28(4):819.

46. Forrest EH, Storey N, Sinha R, Atkinson SR, Vergis N, Richardson P, et al. Baseline neutrophil-to-lymphocyte ratio predicts response to corticosteroids and is associated with infection and renal dysfunction in alcoholic hepatitis. Aliment Pharmacol Ther 2019 -;50(4):442.

\section{Figures}




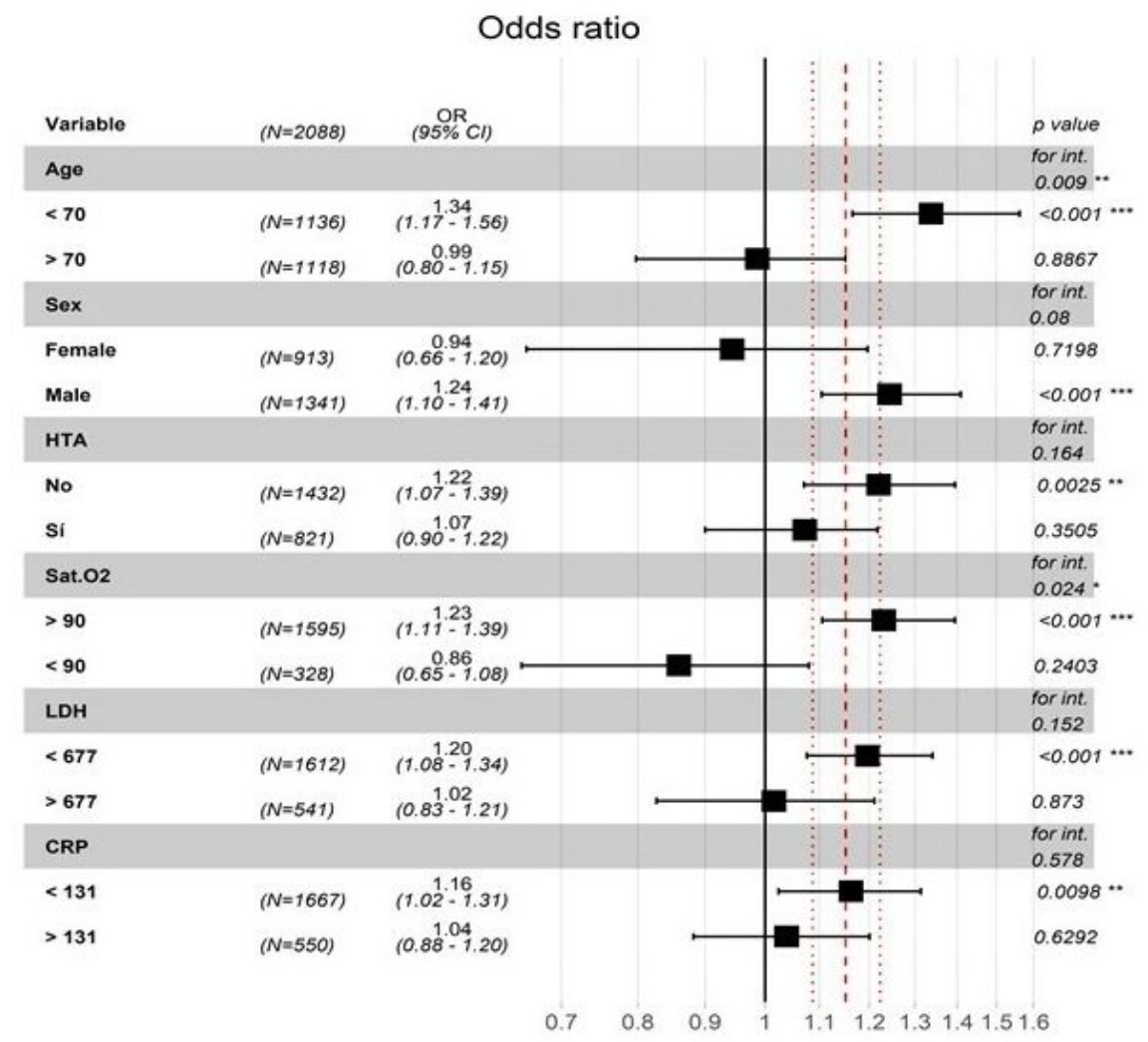

Figure 1

Interactions and stratified analyses for NPR (neutrophil-platelets ratio) adjusted to model A (Table 5) and conducted for age (< 70 and $>70$ years), sex, hypertension (HTA), oxygen saturation (<90 and >90\%) (SatO2), and lactate dehydrogenase (LDH) and C-reactive protein (CRP) both categorized through their respective median values.

\section{Supplementary Files}

This is a list of supplementary files associated with this preprint. Click to download.

- FigS1B.tif

- FigS1C.tif

- FigS1D.tif

- Figs2.tif

- FigS3.tif

- FigS4A.tif

- FigS4B.tif

- FigS5.tif 